\title{
Myocardial bridge and coronary arteries: morphological study and clinical significance
}

\author{
Ashraf Y. Nasr ${ }^{1,2}$ \\ ${ }^{1}$ Department of Anatomy, Faculty of Medicine, Zagazig University, Zagazig, Egypt \\ ${ }^{2}$ Department of Anatomy, Faculty of Medicine, King Abdulaziz University, Jeddah, Kingdom of Saudi Arabia
}

[Received 25 November 2013; Accepted 5 January 2014]

\begin{abstract}
Myocardial bridge $(M B)$ is the myocardial bundles covering a segment of a coronary artery or one of its branches. This work aimed to study the morphological properties of $M B$ and their effects on the structure of the dominant coronary artery. Sixty adult human hearts (40 male and 20 female) were obtained from the Anatomy Department, Faculty of Medicine, King Abdulaziz University. Dissection of the coronary arteries and their main branches was done. Number, site, length and thickness of $M B$ were determined in correlation with coronary dominancy. External diameter of proximal and distal segments of bridged branches and histology of the different segments of the anterior interventricular artery were examined as well. Thirty-six MB were observed in 27 (45\%) hearts (18 male and 9 female). $M B$ were mostly observed on the middle segment of the anterior interventricular artery (52.8\%) and to a lesser extent on its diagonal branch (13.8\%), posterior interventricular artery (13.8\%), median and left marginal branches (5.6\%), and right coronary artery stem (2.8\%). In 30\% out of $56.7 \%$ of right, in $8.3 \%$ out of $26.7 \%$ of balanced and in $6.7 \%$ out of $16.7 \%$ of left dominant hearts revealed $M B$. The mean length of $M B$ was $24.9 \pm 1.98 \mathrm{~mm}$ and that of their thickness was $2.28 \pm 0.13 \mathrm{~mm}$. Morphological differences in external diameter and histological structure of the different parts of bridged branch were observed. Knowledge of morphological aspects and effects of MB provide better therapeutic and surgical interventions for clinicians dealing with patients having MB. (Folia Morphol 2014; 73; 2: 169-182)
\end{abstract}

Key words: coronary arteries, dominancy, myocardial bridge, morphology

\section{INTRODUCTION}

Coronary arteries and their branches course beneath the epicardium within the cardiac adipose tissue. However, at a certain area of their original course, a segment of the coronary artery and/or one of its main branches is/are sometimes partly covered by myocardial tissue. This condition is defined as a myocardial bridges (MB) [22]. MB may constitute a common anatomic variant [18] or a benign congenital abnormalities of coronary arteries $[2,22]$. The prevalence of $\mathrm{MB}$ has been reported to be $15-88.0 \%$ in cadavers and $0.5-12 \%$ by coronary angiography [2, 4, 22]. $\mathrm{MB}$ are commonly seen on the left coronary artery (LCA) trunk and/or on one of its branches, especially

Address for correspondence: Ashraf Y. Nasr, MD, Anatomy Department, King Abdulaziz University, PO Box 80205, Jeddah 21589, Kingdom of Saudi Arabia, tel: +966 (2) 6401000 ext. 20477, fax: +966 (2) 4601000 ext. 20121, e-mail: ashrafnaeem2013@gmail.com

This work has been done in the Anatomy Department, Faculty of Medicine, King Abdulaziz University, Jeddah, Kingdom of Saudi Arabia. 
on the anterior interventricular artery (AIA), which represents $12-63 \%$ of $M B$, its lateral branch (13\%) and its circumflex artery (2.8-6.7\%). Also, they are observed on the diagonal artery (DA), marginal arteries, posterior interventricular artery (PIA) and right coronary artery (RCA) [26].

Although most of MB are clinically silent, the longest and deeper ones may compress coronary vessels underneath and compromise myocardial blood supply. The direct compression of MB during the cardiac systole and enhancement of MB to the natural progression of coronary atherosclerosis with subsequent changes in haemodynamic stress driven by the force of the MB contraction may be the main factors that concomitant the occurrence of coronary heart disease (CHD) with presence of MB $[4,15,22]$. Several studies have also documented the association of MB with CHD such as myocardial ischaemia, arrhythmia, acute coronary syndromes or sudden death $[4,15]$.

From the histopathological and clinical points of view, the presence of MB protects the tunnelled segment of coronary artery from the development of atherosclerosis by 3 main mechanisms, including: low tensile stress, high shear stress and decrease tension of the coronary wall [8]. Thus, the MB are considered as atheroprotective factor through the surrounding myocardium that acts as haemodynamic microenvironment within the bridges [15].

The concept of coronary dominance arose from the fact that the PIA can originate from different arteries. According to Schlesinger [27], the circulation was named 'right dominant' if the artery from which the PIA and posterolateral branches emerge is the RCA, while it was named 'left dominant' if the origin of these branches is the left circumflex artery. The cases in which the RCA gives off the PIA and the left circumflex gives off the posterolateral branches were referred to as having balanced circulation. However, there are other patterns that do not fit into this classification $[12,20]$. Right dominant circulation is the commonest in $42-87 \%$ of the general population, while left dominant circulation for general population is $10-24 \%$ and the co-dominance (balanced) circulation is $3-44 \%[12,20,27]$.

The aim of the present study was to determine the incidence, location, number, length and thickness of the MB over the coronary arteries and/or their main branches in adult human male and female hearts, as well as the relationship between the incidence of MB and dominant circulation of the coronary arteries.
Indeed, the effects of MB on the external diameter of the main branches of coronary arteries and the histological structure of the AIA at the proximal, mid- and distal points of MB were studied.

\section{MATERIALS AND METHODS}

Sixty hearts of adult human cadavers ( 40 males and 20 females) were obtained from the Anatomy Department, Faculty of Medicine, King Abdulaziz University, Jeddah, Saudi Arabia. The hearts were preserved in $10 \%$ formalin. The epicardium and epicardial fat were carefully dissected. The hearts were cleaned with $15 \% \mathrm{KOH}$ solution to extract the remaining pericardial fat. Coronary arteries and their main branches were carefully delineated throughout their courses using a dissecting microscope. The prevalence and location of the MB on the coronary arteries and/or their branches were determined taking sample gender into account. The length, thickness and distance of the MB were measured as well with an electronic (digital) Vernier caliper $(0.01 \mathrm{~mm}$ accuracy). The external diameter of the artery at the points just before and after the MB was also measured. The correlation between incidence of $\mathrm{MB}$ and coronary dominancy, according to Schlesinger [27] dominance criteria (1940) was performed.

Specimens from the AIA at the course points just before, at mid-point and just after the MB were taken. The specimens were prepared for light microscopic examination. 3-5 $\mu \mathrm{m}$ sections from the paraffin blocks of each specimen were stained with haematoxylin and eosin (H\&E), Masson's trichrome (MTC) and Periodic acid Schiff (PAS) stains to determine the effect of MB on the histological structure of different segments of the bridged artery.

The data were collected in excel software sheets and analysed statistically using SPSS version 16 for Windows (Chicago, USA). Mean \pm standard error of mean (SEM) for the continuous variables were calculated, whereas nominal variables were described in terms of number and percentages for sex, male and female. One-way ANOVA and $\chi^{2}$ test (for non-parametrically distributed values) were used for multiple comparisons of the morphological characteristics of $\mathrm{MB}$ among the male and female hearts. Correlation between the length and thickness of $M B$, proximal and distal external diameter of each and both sexes, incidence of $\mathrm{MB}$ and dominancy of coronary circulation were determined by Pearson's correlation coefficient method. Values at $p<0.05$ were considered 

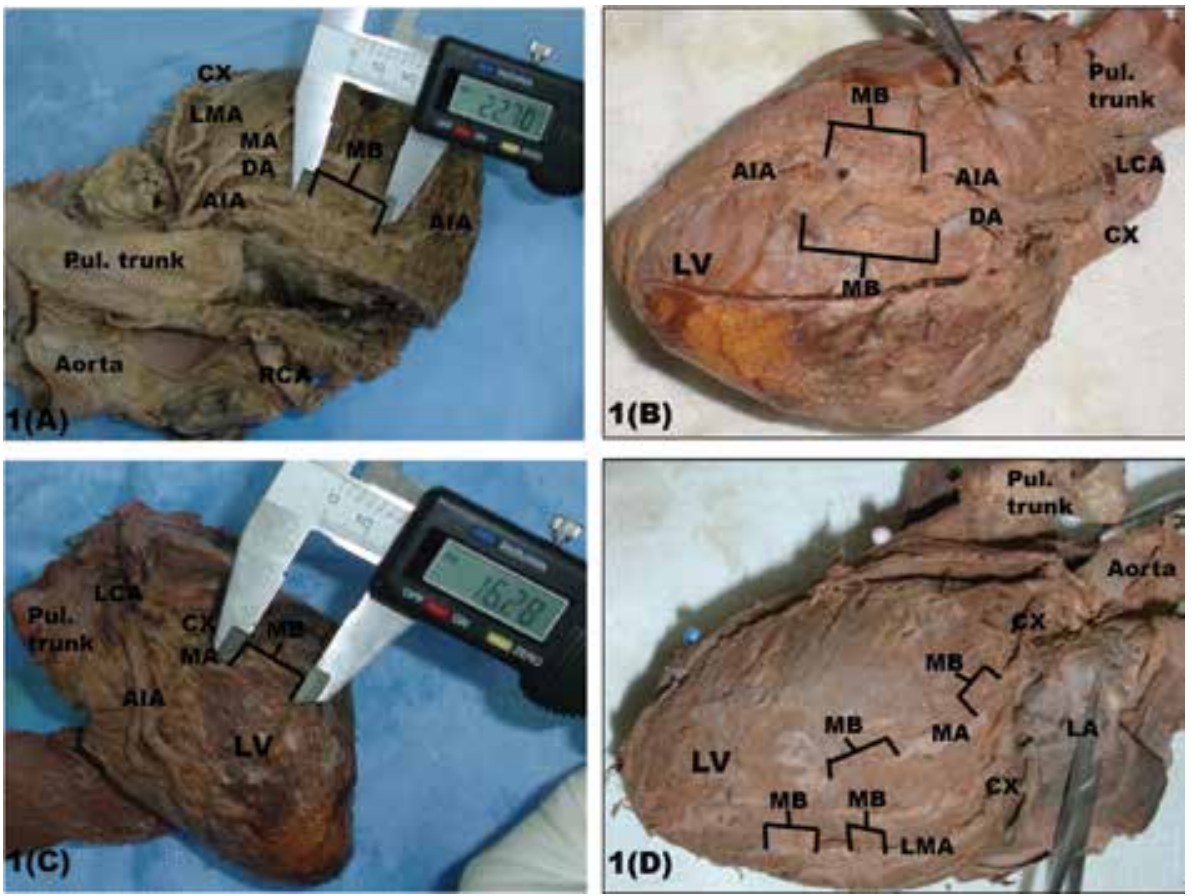

Figure 1. A light photograph of adult human hearts showing the distribution of the myocardial bridges $(\mathrm{MB})$ on the different branches of the left coronary artery (LCA): A. On anterior interventricular artery (AIA); B. On the diagonal branch (DA) of the anterior interventricular artery; C. On the median branch (MA) of the LCA; D. On the left marginal branch (LMA); Cx — circumflex branch of LCA; LA — left auricle; LV — left ventricle; $\mathrm{RCA}$ - right coronary artery.

statistically significant. This descriptive anatomical study was performed after the agreement of the medical ethical committee of the faculty of medicine, King Abdulaziz University, Jeddah, Saudi Arabia.

\section{RESULTS}

A total of $36 \mathrm{MB}$ were identified in 27 out of $60(45 \%)$ hearts, $18(45 \%)$ male and 9 (45\%) female: 20 hearts showed a single $\mathrm{MB}$ ( 12 male and 8 female) affecting 8 AIA, 5 PIA, 2 right marginal arteries (RMA), 2 left marginal arteries (LMA), 2 median branch of LCA and 1 over the stem of RCA, while in 7 out of 27 hearts more than 1 bridge was detected; 5 hearts had $2 \mathrm{MB}$ ( 4 male and 1 female) and 2 male hearts showed $3 \mathrm{MB}$. Thirteen out of $19 \mathrm{MB}$ affecting the AIA were of superficial type and the other $6 \mathrm{MB}$ were of deep type. The number of MB was more frequent in male (26/36) than in those of female (10/36) hearts. Most of MB were noted over the branches of LCA $28 / 36(77.8 \%)$ hearts. The most common location of $M B$ was seen over the AIA, where 19 out of $36 \mathrm{MB}$ $(52.8 \%)$ were observed over the AIA (Fig. 1A). Five (13.8\%) MB were found over the DA of LCA (Fig. 1B), 2 (5.6\%) MB on the median branch of LCA (Fig. 1C), $2(5.6 \%)$ MB over the LMA (Fig. 1D). Eight out of
$36(22.2 \%)$ MB were seen over RCA and its branches (Fig. 2), 5 (13.8\%) over PIA (Fig. 2C), 2 (5.6\%) over the RMA (Fig. 2B) and $1(2.8 \%)$ over the stem of RCA (Fig. 2A). In male hearts, the MB were seen in 18 out of 40 hearts $(45 \%)$. These MB were seen over the AIA in $9 / 40$ (22.5\%) hearts, over the DA in 2/40 (5\%) hearts, over the median branch of LCA in 2/40 $(5 \%)$ hearts, over LMA in $1 / 40(2.5 \%)$ heart, over PIA in $4 / 40(10 \%)$ hearts, over RMA in $1 / 40(2.5 \%)$ heart and over the stem of RCA in $1(2.5 \%)$ heart, while in female, the MB were noted in 9 out of 20 hearts. MB were observed over AIA in 5/20 (25\%) hearts and in $1(5 \%)$ heart over the DA, LMA, PIA and RMA (Table 1).

The MB over AIA were located mainly over upper and middle thirds (Fig. 1), found in $7 / 19$ (36.8\%), over the middle third in $6 / 19$ (31.5\%), over the middle and lower thirds in $4 / 19$ (21\%) and over the lower third only in $2 / 19(10.5 \%)$. In male, in $5(38.5 \%)$ of the MB were seen over the upper and middle thirds, in 4 (30.8\%) over the middle third, in $3(23.1 \%)$ over the middle and lower thirds and in $1(7.7 \%)$ over the lower third. However, in female hearts, 2 (33.3\%) of the MB were seen over the upper and middle thirds, 2 (33.3\%) over middle third, $1(16.7 \%)$ over both middle and lower thirds and 1 (16.7\%) over its lower third (Table 2). 

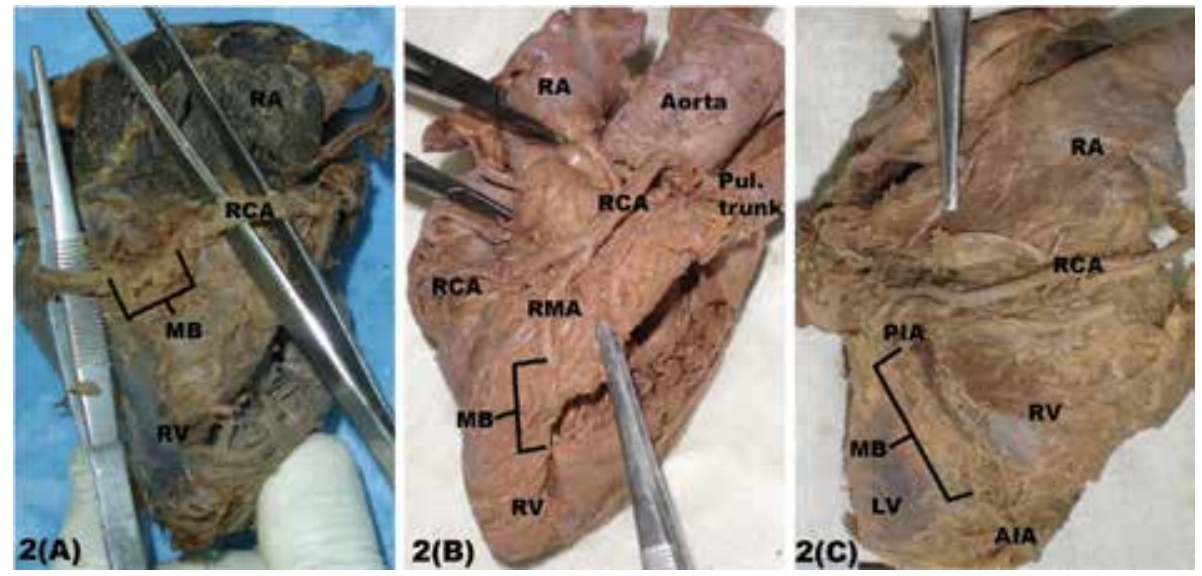

Figure 2. A light photograph of adult human hearts showing distribution of the myocardial bridges (MB) on the different branches of the right coronary artery (RCA): A. On the main stem of right marginal artery (RMA); B. On the RMA of RCA; C. On the posterior interventricular artery (PIA); AIA — anterior interventricular artery; LV — left ventricle; RV — right ventricle; RA — right auricle; LA — left atrium.

Table 1. Incidence of myocardial bridges (MB) on the different branches of coronary arteries

\begin{tabular}{|c|c|c|c|c|c|c|}
\hline \multirow[t]{2}{*}{ Site of MB } & \multicolumn{2}{|c|}{ Total MB } & \multicolumn{2}{|c|}{ Male hearts (40) } & \multicolumn{2}{|c|}{ Female hearts (20) } \\
\hline & $\begin{array}{l}\text { Relative } \\
\text { (36 MB) }\end{array}$ & $\begin{array}{c}\text { Absolute } \\
\text { (27 hearts) }\end{array}$ & $\begin{array}{l}\text { Relative } \\
\text { (26 MB) }\end{array}$ & $\begin{array}{l}\text { Absolute } \\
\text { (18 hearts) }\end{array}$ & $\begin{array}{l}\text { Relative } \\
\text { (10 MB) }\end{array}$ & $\begin{array}{l}\text { Absolute } \\
\text { (9 hearts) }\end{array}$ \\
\hline AIA & $19(52.8 \%)$ & $14(23.3 \%)$ & $13(50 \%)$ & $9(22.5 \%)$ & $6(60 \%)$ & $5(25 \%)$ \\
\hline DA & $5(13.8 \%)$ & $3(5 \%)$ & $4(15.4 \%)$ & $2(5 \%)$ & $1(10 \%)$ & $1(5 \%)$ \\
\hline LMA & $2(5.6 \%)$ & $2(3.3 \%)$ & $1(3.8 \%)$ & $1(2.5 \%)$ & $1(10 \%)$ & $1(5 \%)$ \\
\hline MA & $2(5.6 \%)$ & $2(3.3 \%)$ & $2(7.7 \%)$ & $2(5 \%)$ & 0 & 0 \\
\hline PIA & $5(13.8 \%)$ & $3(5 \%)$ & $4(15.4 \%)$ & $2(5 \%)$ & $1(10 \%)$ & $1(5 \%)$ \\
\hline RMA & $2(5.6 \%)$ & $2(3.3 \%)$ & $1(3.8 \%)$ & $1(2.5 \%)$ & $1(10 \%)$ & $1(5 \%)$ \\
\hline RCA Stem & $1(2.8 \%)$ & $1(1.7 \%)$ & $1(3.8 \%)$ & $1(2.5 \%)$ & 0 & 0 \\
\hline Total MB & $36(100 \%)$ & 27 (45\%) & $26(100 \%)$ & $18(45 \%)$ & $10(100 \%)$ & $9(45 \%)$ \\
\hline
\end{tabular}

AIA — anterior interventricular artery; DA — diagonal branch of AIA; LMA — left marginal artery; MA — median branch of left coronary artery; PIA — posterior interventricular artery; RMA — right marginal artery; RCA — right coronary artery

Table 2. Distribution of myocardial bridges over different parts of anterior interventricular artery

\begin{tabular}{lcccc}
\hline & Apper and middle thirds & Middle third & Middle and lower thirds & Lower third \\
\hline Male & $5(38.5 \%)$ & $4(30.8 \%)$ & $3(23.1 \%)$ & $1(7.7 \%)$ \\
Female & $2(33.3 \%)$ & $2(33.3 \%)$ & $1(16.7 \%)$ & $1(16.7 \%)$ \\
Total & $7(36.8 \%)$ & $6(31.5 \%)$ & $4(21 \%)$ & $2(10.5 \%)$ \\
\hline
\end{tabular}

The MB were seen at a mean distance of $38.2 \pm$ $\pm 5.3 \mathrm{~mm}$ from the beginning of the AIA with a range of $20.9-86.7 \mathrm{~mm}$ from the same point. The mean of MB length was $24.9 \pm 1.98 \mathrm{~mm}$ and that of MB over AlA was $26.8 \pm 3.5 \mathrm{~mm}$ in male and $26.5 \pm 3.4 \mathrm{~mm}$ in female hearts. The mean of the MB thickness was $2.28 \pm 0.13 \mathrm{~mm}, 2.5 \pm 0.17 \mathrm{~mm}$ over AIA, $2.37 \pm 0.22 \mathrm{~mm}$ over AIA in male and $2.37 \pm 0.24 \mathrm{~mm}$ over AIA in female, $1.8 \pm 0.3 \mathrm{~mm}$ over DA, $1.41 \pm 0.24 \mathrm{~mm}$ over PIA, $1.46 \pm 0.21 \mathrm{~mm}$ over LMA and $0.99 \pm 0.15$ over RMA. The MB showed little effect on the diameter of their underlying arteries, where the mean of the external diameter of AIA proximal to the $\mathrm{MB}$ was $3.1 \pm 0.16 \mathrm{~mm}$ and its measure was $2.97 \pm 0.16$ distal to the $M B$, for $D A$ measured $2.52 \pm 0.13$ proximal to $\mathrm{MB}$ and $2.42 \pm$ $\pm 0.13 \mathrm{~mm}$ distal to $\mathrm{MB}$, for PIA was $2.56 \pm 0.24 \mathrm{~mm}$ proximal to $\mathrm{MB}$ and $2.44 \pm 0.18 \mathrm{~mm}$ distal to $\mathrm{MB}$, 
Table 3. Measurements of myocardial bridges $(\mathrm{MB})$ and diameters of their underlies arteries (mean $\pm \mathrm{SEM}$ )

\begin{tabular}{lccccc}
\hline Coronary artery branch & Length of MB & $\begin{array}{c}\text { Thickness } \\
\text { of MB }\end{array}$ & $\begin{array}{c}\text { Distance from } \\
\text { the origin }\end{array}$ & $\begin{array}{c}\text { Pre-bridge } \\
\text { diameter (D1) }\end{array}$ & $\begin{array}{c}\text { Post-bridge } \\
\text { diameter (D2) }\end{array}$ \\
\hline Total MB & $24.9 \pm 1.98^{\mathrm{a}}$ & $2.28 \pm 0.13^{\mathrm{a}}$ & $38.1 \pm 4.4$ & - & - \\
Total AIA & $26.7 \pm 2.9^{\mathrm{b}}$ & $2.5 \pm 0.17^{\mathrm{b}}$ & $38.2 \pm 5.3$ & $3.09 \pm 0.16$ & $2.97 \pm 0.16$ \\
Male AIA & $26.8 \pm 3.5^{\mathrm{c}}$ & $2.37 \pm 0.22^{\mathrm{c}}$ & $44.5 \pm 2.5$ & $3.2 \pm 0.28$ & $3.02 \pm 0.12$ \\
Female AIA & $26.5 \pm 3.4^{\mathrm{d}}$ & $2.37 \pm 0.24^{\mathrm{d}}$ & $29.8 \pm 2.4$ & $3.04 \pm 0.2^{\mathrm{n}}$ & $2.94 \pm 0.2 \mathrm{n}$ \\
Total DA & $6.5 \pm 0.9$ & $1.8 \pm 0.3$ & - & $2.52 \pm 0.13$ & $2.42 \pm 0.13$ \\
Male DA & $7.25 \pm 1.1^{\mathrm{e}}$ & $1.7 \pm 0.3^{\mathrm{e}}$ & - & $2.54 \pm 0.16$ & $2.42 \pm 0.14$ \\
Female DA & $5.0 \pm 1.0$ & $1.95 \pm 0.55$ & - & $2.48 \pm 0.34$ & $2.42 \pm 0.36$ \\
PIA & $5.13 \pm 0.31^{\dagger}$ & $1.41 \pm 0.24^{\dagger}$ & - & $2.56 \pm 0.24$ & $2.44 \pm 0.18$ \\
LMA & $9.0 \pm 4.0$ & $1.46 \pm 0.21$ & - & $2.3 \pm 0.007^{\mathrm{H}}$ & $2.2 \pm 0.005^{\mathrm{H}}$ \\
RMA & $10.0 \pm 2.0^{\mathrm{g}}$ & $0.99 \pm 0.15^{\mathrm{g}}$ & - & $2.05 \pm 0.15$ & $1.99 \pm 0.15$ \\
\hline
\end{tabular}

Number (60); mean \pm SEM, a, b, c $d, e, f, g, H, n$ : an extreme significance between $M B$ length vs. MB thickness of same group $(p<0.0001)$; AIA - anterior interventricular artery; DA — diagonal branch of AIA; PIA — posterior interventricular artery; LMA — left marginal artery; RMA — right marginal artery

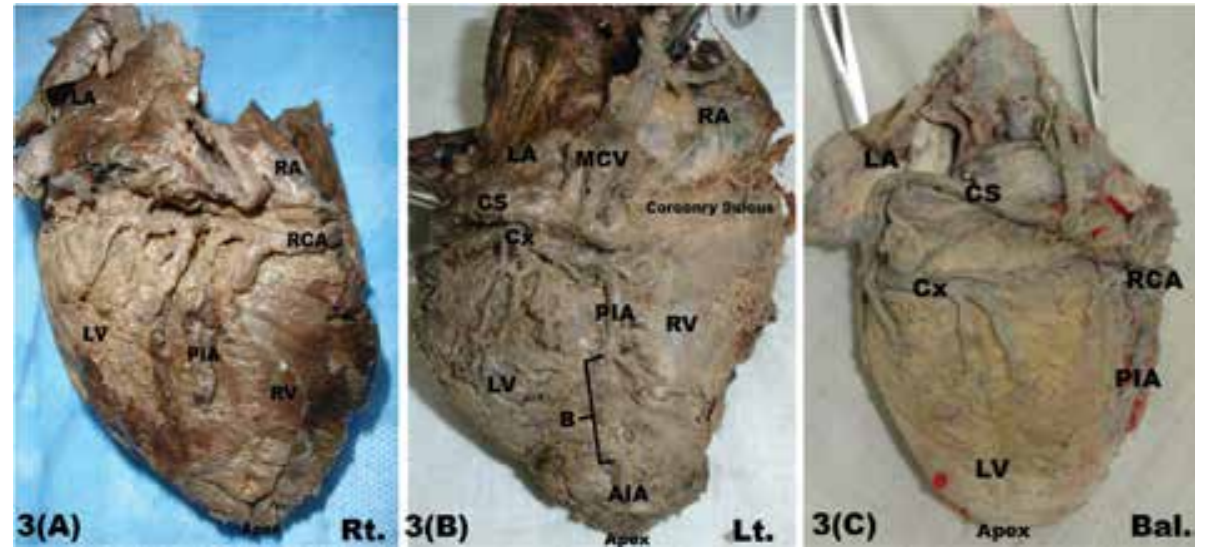

Figure 3. A light photograph of adult human hearts showing 3 types of dominant coronary circulation: A. Right dominant type; B. Left dominant type; C. Balanced type; AIA — anterior interventricular artery; RCA — right coronary artery; LV — left ventricle; PIA — posterior interventricular artery; RV — right ventricle; RA — right auricle; CS — coronary sinus; MCV — middle cardiac vein; CX — circumflex branch of left coronary artery; LA — left auricle; MB — myocardial bridge.

for LMA it measured $2.3 \pm 0.007$ proximal to $\mathrm{MB}$ and $2.2 \pm 0.005$ distal to $\mathrm{MB}$ and it was $2.05 \pm 0.15 \mathrm{~mm}$ proximal and $1.99 \pm 0.15 \mathrm{~mm}$ distal to $\mathrm{MB}$ for RMA. Statistically, an extreme statistical significance ( $p<0.0001$ ) was detected between the length and thickness of MB over most of the branches. Meanwhile, the pre-bridge and post-bridge diameters of most of the arterial branches reported no significance in same group or between male and female hearts (Table 3 ).

Three main patterns of the coronary circulation (Fig. 3) were observed in the present study (Table 4). Right coronary dominancy (Fig. 3A) was the predominant pattern in 34 out of 60 (56.7\%) hearts with no sex difference, where it was found in 23 out of 40 male hearts (57.5\%) and in 11 out of 20 female hearts
(55\%). Meanwhile, the balanced coronary circulation (Fig. 3C) was noted in 16 out of 60 hearts (26.7\%). This pattern was observed in 11 out of 40 male hearts $(27.5 \%)$ and was found in 5 out of 20 female hearts (25\%). Left coronary circulation (Fig. 3B) was seen in $10(16.7 \%)$ hearts - $6(15 \%)$ male and $4(20 \%)$ female (Table 4).

In correlation with the dominancy of coronary circulation, most of $\mathrm{MB}$ were observed in right dominant hearts, where they were observed in 18 out of 34 hearts $(52.9 \%) ; 12$ out of 23 were male (52.2\%) and 6 out of 11 were female (54.6\%) hearts. Meanwhile, MB were seen in 4 out of 10 hearts (40\%) of the left coronary dominancy, 3/6 male (50\%) and $1 / 4(25 \%)$ female hearts. In the hearts with balanced 
Table 4. Dominant coronary circulation and its correlation with the incidence of myocardial bridge (MB)

\begin{tabular}{|c|c|c|c|c|c|c|c|c|c|}
\hline \multirow[t]{2}{*}{ Variable } & \multicolumn{3}{|c|}{ Right dominancy } & \multicolumn{3}{|c|}{ Left dominancy } & \multicolumn{3}{|c|}{ Balanced dominancy } \\
\hline & Male & Female & Total & Male & Female & Total & Male & Female & Total \\
\hline Incidence (\%) & $57.5 \%$ & $55 \%$ & $56.7 \%$ & $15 \%$ & $20 \%$ & $16.7 \%$ & $27.5 \%$ & $25 \%$ & $26.7 \%$ \\
\hline With MB (Absolute) & $30 \%$ & $30 \%$ & $30 \%$ & $7.5 \%$ & $5 \%$ & $6.7 \%$ & $7.5 \%$ & $10 \%$ & $8.3 \%$ \\
\hline With MB (Relative) & $52.2 \%$ & $54.6 \%$ & $52.9 \%$ & $50 \%$ & $25 \%$ & $40 \%$ & $27.3 \%$ & $40 \%$ & $31.3 \%$ \\
\hline MB on LCA (Relative) & $34.7 \%$ & $27.3 \%$ & $32.3 \%$ & $33.3 \%$ & $25 \%$ & $30 \%$ & $27.3 \%$ & $40 \%$ & $31.3 \%$ \\
\hline MB on RCA (Relative) & $17.3 \%$ & $18.3 \%$ & $17.6 \%$ & $16.7 \%$ & $25 \%$ & $10 \%$ & $9.1 \%$ & 0 & $6.3 \%$ \\
\hline
\end{tabular}

LCA — left coronary artery; RCA — right coronary artery; Absolute — related to the total number of hearts in each group; Relative — related to the incidence of myocardial bridges in the same group
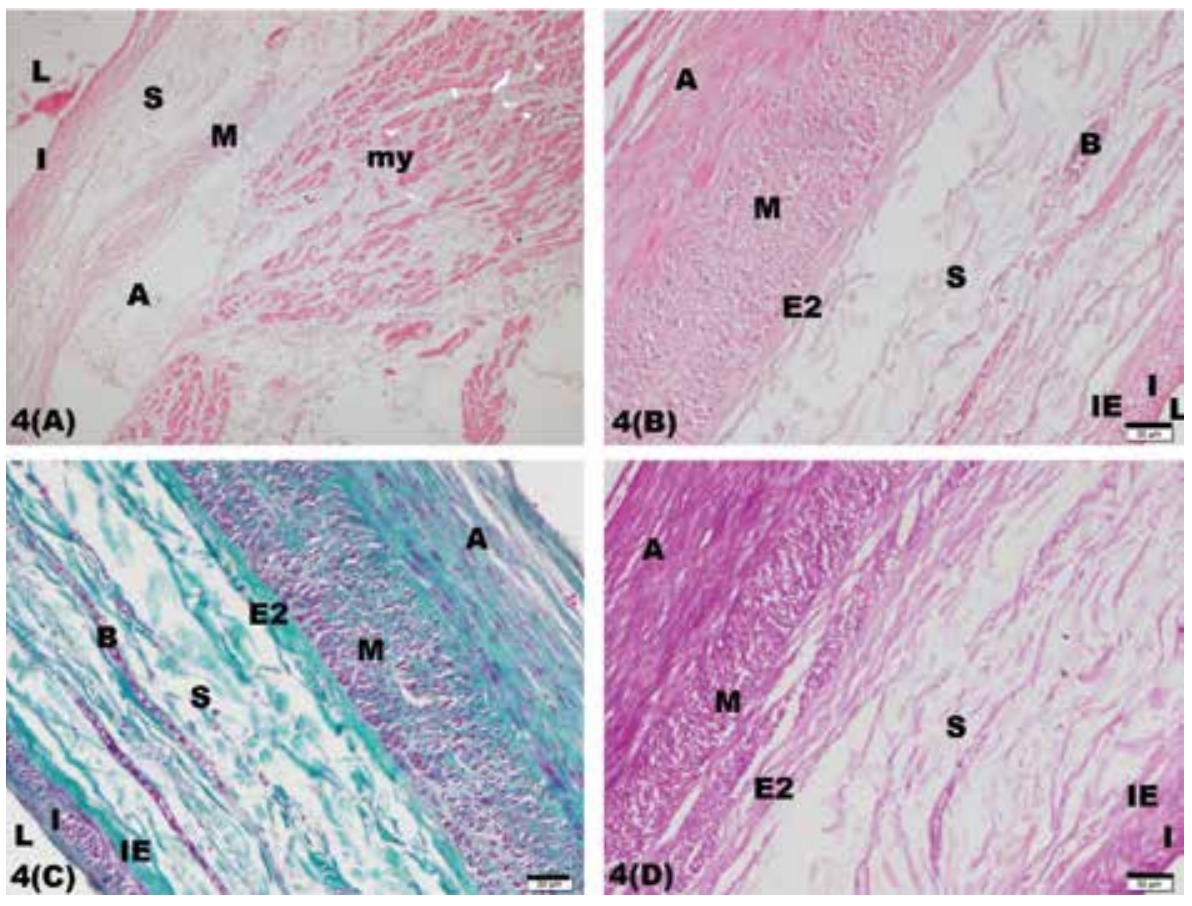

Figure 4. Light photomicrographs of the proximal segment of anterior interventricular artery to the myocardial bridge showing in 4 (A, B): wide arterial lumen (L), thick intimal (I) and medial (M) layers (A) with wide sub-intimal layer (S) in-between. Direct contact is seen between the outer adventitial layer (A) of the artery and its underlying myocardial fibres (my); C. An excessive amount of the collagen fibres is seen within the sub-intimal layer (S) and adventitia (A) with duplication of the internal elastic lamina into inner (IE) and outer (E2) layers by the masson trichrome stained section; $\mathbf{D}$. A positive PAS reaction is present with the different layers of the segmental wall; $\mathrm{B}$ - blood vessel; panel $A$ - H\&E $\times$ 40; panel B - H\&E $\times 100$; panel C — Masson trichrome stain $\times 100$; panel D — PAS stain $\times 100$.

circulation, MB were seen in 5 out 16 hearts (31.3\%), $3 / 11(27.3 \%)$ were male and $2 / 5(40 \%)$ were female hearts (Table 4).

The structure of the bridged AIA revealed different histological findings in its proximal, under and distal segments. The segment of AIA proximal to MB showed wide lumen, thick intima and sub-intimal layers, prominent muscle fibres within its media with no well demarcation between the adventitia of the artery and its surrounding myocardium (Fig. 4A). An excessive amount of the elastic fibres was determined within the sub-inti- mal layer with duplication of the internal elastic lamina, in-between the muscle bundles of the media and within the adventitia (Fig. 4B, C). However, the segment of AIA under the MB revealed relative narrower lumen and thinner intimal and medial layers with no direct contact between the adventitia and muscle fibres of its overlying $M B$ (Fig. 5A-C). In the distal segment of the affected AIA, a relative wider lumen and thicker intimal and medial layers were observed. Its adventitia contained nerve, collagen and elastic fibres as well (Fig. 6A-C). 

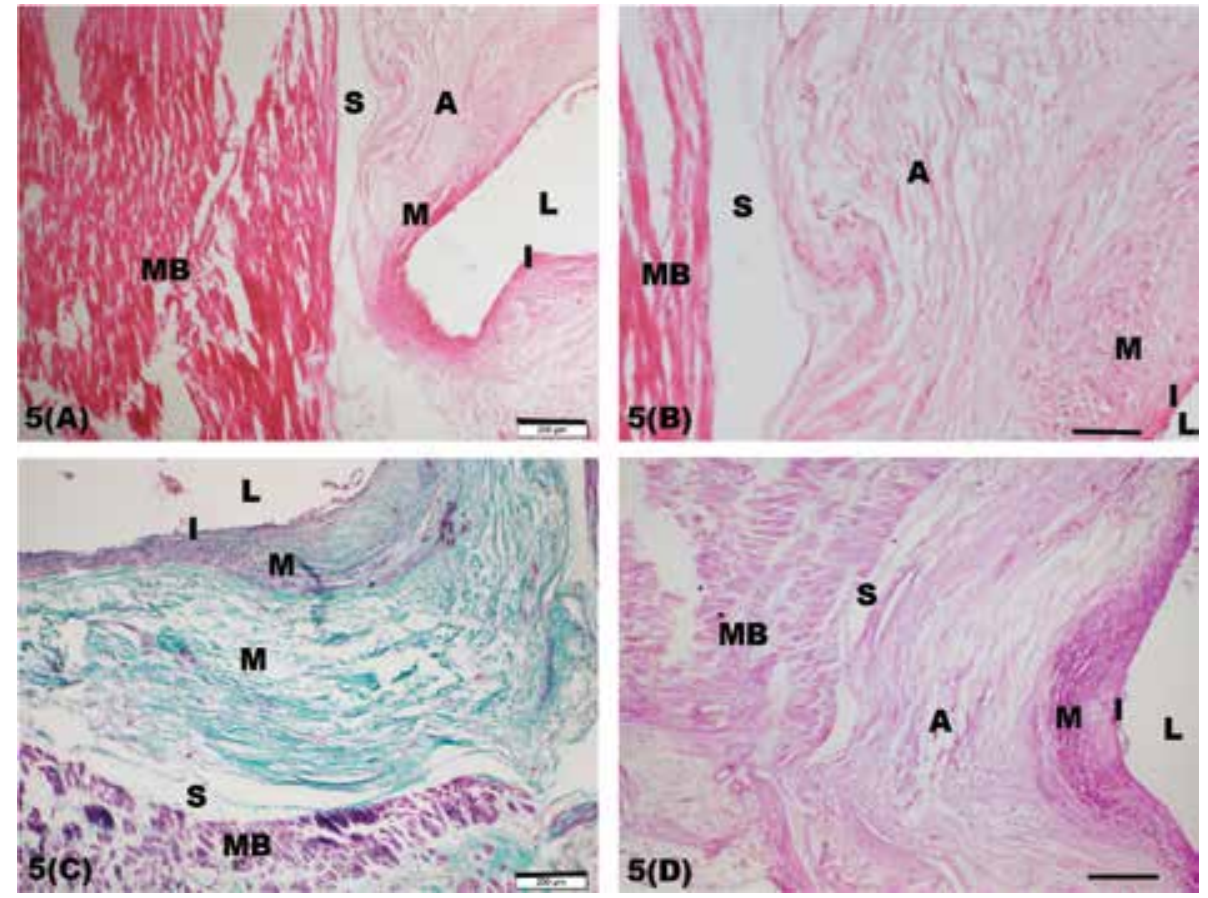

Figure 5. Light photographs of the bridged segment of the anterior interventricular artery showing in $5(\mathbf{A}, \mathbf{B})$ thin intimal (I) and medial (M) layers and thick adventitia $(A)$ with no direct contact between the adventitial layer $(A)$ and its overlying myocardial bridge $(M B)$, as there is a well demarcated space $(\mathbf{S})$ in-between; $\mathbf{C}$. Little amount of collagen fibres is seen within the sub-intimal layer and in-between the muscle fibres of MB, while the adventitia contains an excessive amount of collaged fibres; D. A strong positive PAS reaction is seen in the intimal layer (I) and a weak reaction is observed in the other layers of the arterial wall; $\mathrm{L}$ - the lumen of arterial segment; panels $A$ and $B-\mathrm{H} \& \mathrm{E}$; panel C - Masson trichrome stain $\times 100$; panel $D$ - PAS-stain $\times 100$.
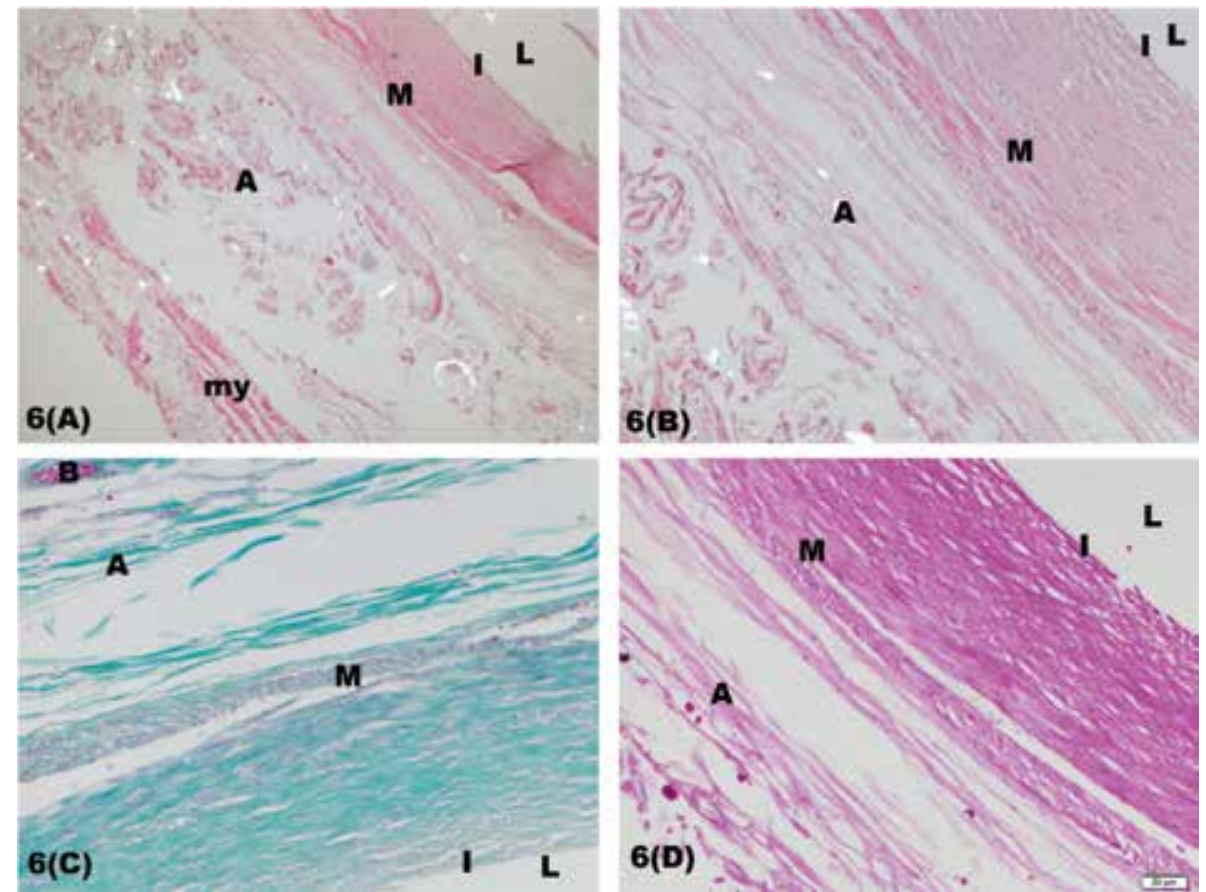

Figure 6. Light micrographs of the distal segment of anterior interventricular artery to myocardial bridge showing a relative wide lumen (L), thick intima (I) and a moderate thick media (M) with little amount of collagen fibres in intimal (I) and sub-intimal layers of this segment. Weak positive PAS reaction is observed in intimal (I), sub-intimal and adventitial (A) layers of this segment; B - blood vessels; my — myocardial muscle fibres; panel $A-H \& E \times 40$; panel $B-H \& E \times 100$; panel $C$ - Masson trichrome stain $\times 100$; panel D - PAS stain $\times 100$. 


\section{DISCUSSION}

MB are currently controversial research subject. Although the MB were recognised many years ago, their exact physiopathology and clinical significance have not yet been definitely established till now. Different explanations were supposed to explain the evolution and physiopathology of MB. Some authors considered MB as being normal intramural arteries [21]; others believed $\mathrm{MB}$ as a vascular heart variation that resulted from failure of synchronisation between the development of the myocardium and the epicardial coronary branches, where a segment of 1 epicardial branch was tunnelled by a bundle of myocardium [2,5]; conversely, other authors have suggested that $M B$ are not an anatomic variation, but they occurred as a result of a developmental defect in the resorption of the cardiac musculature that encircles the epicardial arteries. Lastly, on the developmental basis, 3 types of coronary arteries were mentioned. In type 1 , the major coronary arteries were embedded in the myocardium beneath the epicardial surface. This type was described in rodents. In type 2, coronary arteries had a predominant epicardial course. This type includes human, carnivores, small ruminants and primates. In type 3, extremely rare MB were reported in animals such as horse and pig. Thus, the $\mathrm{MB}$ are congenital in origin and likely reflect an evolutionary remnant in genetic code [22].

In the present study, the incidence of MB revealed no sex difference in the number of the affected hearts, where 18 out of $40(45 \%)$ male hearts and 9 out of $20(45 \%)$ female hearts showed MB. Meanwhile, a male predominance was observed regarding the number of $M B$, as $26 \mathrm{MB}$ were recorded in 40 male hearts and $10 \mathrm{MB}$ were observed in $20 \mathrm{fe}-$ male hearts. In agreement with the results of the present study, MB were noted in $40.3 \%$ and $40.4 \%$ of their specimens respectively $[5,28]$. However, a higher incidence of MB $(64 \%, 56 \%, 55.6 \%$ and $52 \%)$ was reported in literature $[6,12]$. Moreover, a significance discrepancy was reported between cadaveric and angiographic studies regarding the incidence, frequency and prevalence of $M B$, where in cadaveric studies, the range of $M B$ incidence was $5-86 \%$, while in the angiographic studies, the occurrence of $\mathrm{MB}$ ranged from $0.5 \%$ to $33 \%[4,19$, $26]$. The difference in the incidence and frequency of $M B$ was supposed in part to be attributed to the sample size, morphological characters of $M B$, method of research, gender and ethnicity of the population selected [26].

In the present study, the most common location of the MB was seen over the AIA branch (52.8\%) of the LCA, especially its middle third, with no significant sex difference. These findings were in accordance with the results stated in literature $[5,12,26,28]$. According to the results of previous literature, the second common location of the MB was the diagonal branches of AIA and PIA branch of RCA (13.8\%, each) [5]. However, a lower incidence of MB was reported over DA and PIA branches $[12,28]$. The actual cause of higher incidence of the MB over middle third of the AIA remains unclear [6].

In the present study, single MB was seen in 20 out of 27 hearts $(74.1 \%)$, double MB in 5 hearts $(18.5 \%)$ and $3 \mathrm{MB}$ in 2 hearts (7.4\%). However, Fazliogullari et al. [12] found $55 \mathrm{MB}$ in 32 out of 50 hearts, $18(56.3 \%)$ single, 8 (25\%) double, $4(12.5 \%)$ triple and more than $3 \mathrm{MB}$ in $6.4 \%$. Meanwhile, Saidi et al. [28] in Kenyans found single MB in 33 out of 44 (75\%) of the affected hearts, double MB in $2(4.5 \%)$ hearts and triple or more in $5(20.5 \%)$ hearts. Ballesteros et al. [5] observed $92 \mathrm{MB}$ in 62 out of 154 hearts, single $M B$ in $42(67.8 \%)$ hearts, $2 \mathrm{MB}$ in 11 (17.7\%) hearts, $3 \mathrm{MB}$ in $8(12.9 \%)$ hearts and $4 \mathrm{MB}$ in $1(1.6 \%)$ heart.

In accordance with the results of previous cadaveric studies in Colombian [5] and Kenyan [28] populations, the result of present study revealed higher incidence of MB in male hearts (72.2\%) than those of female hearts $(27.8 \%)$, with no significant statistical difference. However, a female predominance was reported by Konen et al. [18]. Thus, the higher incidence of CHD in males than those in females might be in part related to the higher incidence of MB in male hearts than that of female hearts.

Morphological characters of $\mathrm{MB}$, including number, location, length, thickness and distance from the origin of the bridged artery might have an effective role in producing ischaemic symptoms [4]. In literature, a wide range of $\mathrm{MB}$ length was reported, where their length varied from $3.6 \mathrm{~mm}$ to more than $50 \mathrm{~mm}$ [19]. In the present study, the mean length of $\mathrm{MB}$ was $24.9 \pm 1.98 \mathrm{~mm}$ for all $\mathrm{MB}, 26.7 \pm 2.9 \mathrm{~mm}$ over AlA, $6.5 \pm 0.9 \mathrm{~mm}$ over DA and $5.13 \pm 0.13 \mathrm{~mm}$ over PIA, however, the mean length of MB was $19.4 \pm 10.4 \mathrm{~mm}$ in Colombians [5]. In agreement with the results of the present study, the mean length of $M B$ was $30,26,25 \mathrm{~mm}$ and $22.7 \pm 11.94 \mathrm{~mm}[19,20,28]$. In $11 \%$ of the cases, the MB revealed an average 
length of 34.9-50 $\mathrm{mm}$ [28], whereas lower averages (13-17 $\mathrm{mm}$ ) were reported $[5,12]$. The length of MB was significantly higher in LCA branches than those in RCA. Such longer MB might predispose to myocardial ischaemia in absence of atheroma due to prolonged systolic compression [20].

The difference in prevalence rate of MB could be explained to some extent by studying the developmental aspects of coronary arteries, where in an earlier stage of cardiogenesis the tubular heart consists of an endothelial tube within a muscular tube with no epicardium. In a later stage, the septum transversum gives proepicardial outgrowth that forms the epicardium. The cells of the proepicardial migrate to envelop the heart to form the epicardium and pericardium. The migration of epicardial cells is regulated by signalling mechanisms that in turn give out the signals participating in regulation of myocyte proliferation, growth and final myocardial pattern. The myocardium gives factors to control the epithelial-mesenchymal transition of epicardial cells. The mesenchymal cells within the myocardium form the coronary arterial system. The migration of mesenchymal cells through the developing myocardium could explain the embryogenesis of MB over the branches of coronary arteries [25].

In the previous cadaveric studies, thickness of $\mathrm{MB}$ varied from 0.18 to $3.8 \mathrm{~mm}$. The thickest $M B$ were seen over AIA and the thinnest ones were noted over LMA [5]. However, a wider range (5-27 mm) and a higher mean (12 mm) of MB thickness were noted [20]. The later authors added that in $91.3 \%$ of the cases the tunnelled coronary arteries exhibited deep intramural course, while in the remaining $(8.6 \%)$ cases, the tunnelled coronary arteries had a superficial intramural course. These superficial MB were found over the AIA. The results of the present study revealed no significant gender differences in the length, thickness or location of MB. In Kenyans, the $\mathrm{MB}$ were classified depending on their thickness into 3 main categories: 1) superficial, which had a range of thickness $0.3-1.87 \mathrm{~mm}$ in $68.3 \%$ of cases; 2) intermediate, with a range of thickness $1.88-3.45 \mathrm{~mm}$ in $22.7 \%$ of cases and 3 ) deep, with a range $3.46-$ $-5 \mathrm{~mm}$ in $9 \%$ of cases. The author revealed no significant correlation between the length and depth of MB [28]. In Turkish, Fazliogullari et al. [12] stated that the range of $M B$ thickness was $0.18-3.29 \mathrm{~mm}$ and its mean was $0.88 \pm 0.59 \mathrm{~mm}$ for all $\mathrm{MB}, 1.7 \pm$ $\pm 0.9 \mathrm{~mm}$ in deep $\mathrm{MB}$ and $1.0 \pm 0.9 \mathrm{~mm}$ in superficial type, with a high significant correlation in-between. In the present study, the mean thickness of MB was $2.28 \pm 0.13 \mathrm{~mm}$ for all, $2.5 \pm 0.17$ for those over AIA, $1.8 \pm 0.3 \mathrm{~mm}$ for those on DA, $1.41 \pm 0.24 \mathrm{~mm}$ for MB over PIA and $0.99 \pm 0.15$ for MB on RMA. Moreover, a high significant correlation was noted ( $p<0.001$ ) between the length and thickness of MB of the same group.

Depending on the course of AIA and the direction of the overlying myocardial fibres, MB were classified into superficial and deep [10]. The authors added that in superficial MB the AIA passed within the interventricular sulcus and was crossed by the myocardial fibres perpendicular or in acute angle, while in deep form the AIA deviated towards the right ventricle. Moreover, the authors found deep MB on AIA in $24.4 \%$ of the cases. In accordance with the results of the later study, Fazliogullari et al. [12] reported that the superficial type of $M B$ was seen in $77.4 \%$ of $31 \mathrm{MB}$ on AIA and the deep type was found in $22.6 \%$ of these cases. In the present study, 5 out of 19 MB (26.3\%) on AIA were of deep type and the remaining (73.7\%) was of superficial type. Thus, most of MB were of superficial type. For this reason, most of $\mathrm{MB}$ were asymptomatic and did not cause ischaemic manifestations. Moreover, a third type of MB was described into which the tunnelled artery was covered partially by myocardial fibres and partly by fatty, fibrous, or nervous tissue [5]. In addition, 4 types of MB were described: superficial, deep, incomplete and subendocardial $[10,18]$.

In agreement with the results of the present study, a significant correlation was exhibited between the length and thickness of MB [4, 12]. Moreover, Bourassa et al. [4] added that with left ventricle hypertrophy, length and thickness of MB were predisposing factors to myocardial ischaemia. Thus, the length and thickness of MB might have an effect on the ischaemic manifestations of the heart. Conversely, no significant correlation was found between the length and thickness of $M B$, as well as no sex or ethnicity difference in prevalence of length, thickness or location of MB were observed $[20,28]$.

In literature, the distance between the beginning of $\mathrm{MB}$ and the original point of the affected artery was rarely reported $[12,28]$. In the present study, the mean distance between the beginning point of $\mathrm{MB}$ and that of AIA was $38.2 \pm 5.3 \mathrm{~mm}$ and its range was 20.9-86.7 $\mathrm{mm}$ with a significant difference be- 
tween them. In male hearts, the mean of this distance was $44.5 \pm 2.5 \mathrm{~mm}$, while in female hearts it was $29.8 \pm 2.4 \mathrm{~mm}$. In disagreement with the results of the present study, the mean of this distance measured $30.08 \pm 15.51 \mathrm{~mm}$ and its range was $0-85.7 \mathrm{~mm}$ in Turkish hearts, but without sex difference [12]. Moreover, in Kenyans, MB on AIA were found at a mean distance of $43.51 \pm 12.24 \mathrm{~mm}$ with a range of 10-70 $\mathrm{mm}$ from the coronary sinus with no significant difference between male $(43.63 \pm 11.85 \mathrm{~mm})$ and female $(43.18 \pm 11.67 \mathrm{~mm})$ hearts [28]. In Japanese, the distance of MB from the beginning of AIA was $48 \pm 12 \mathrm{~mm}$ in patients with myocardial infarction (MI) and $45.7 \pm 13 \mathrm{~mm}$ in patients without $\mathrm{Ml}$ with a range of 15-100 $\mathrm{mm}$ [15].

The concept of dominance coronary circulation arose from the fact that the PIA can originate from different arteries, what was first proposed by Schlesinger [27], who stated that the dominant coronary circulation was named 'right dominant' if the artery from which the PIA and posterolateral branches emerge was the RCA, while it was named 'left dominant' if the origin of these branches was the left circumflex artery. The cases in which the RCA gives off the PIA and the left circumflex gives off the posterolateral branches were referred to as having balanced circulation. The most prevalent pattern in normal human hearts was the RCA dominance that was found in $42-90 \%$ of individuals, while the incidence of the LCA dominance was reported in different series varying from $8 \%$ to $33 \%$ [20].

According to the criteria introduced by Schlesinger [27], for determination of the dominant coronary arterial circulation, the right coronary dominance was found in $56.7 \%$ of hearts, followed by the balanced coronary artery dominance in $26.7 \%$ of hearts and left coronary dominance in $16.7 \%$ of hearts with no significant sex difference in the present study. This was consistent with many of the published studies $[13,20,27]$. However, the range of right dominance was $87-62.5 \%$ [5]. Meanwhile, the incidence of the balanced coronary dominance in the present study was (26.7\%) higher than the reported ranges of $3-19.1 \%$ in literature $[5,19,20]$, in the other hand, its value was lower than $34 \%$ and $44 \%$ that were reported by Schlesinger [27] and Fazliogullari et al. [12], respectively. An approximate incidence (15-20\%) to that of the left dominance in the present study $(16.7 \%)$ was reported [13, 27], while a lower incidence was found in other studies
[5] and a higher incidences (33\% and 23\%) were previously reported as well [20].

The variant incidence of dominant coronary circulation may be related in part to the ethnic diversity of the populations under the study, number of specimens, type of study (cadaveric or radiologic) or due to the classification pattern of coronary circulation [20], where the right coronary dominance were observed in $82 \%$ to $84.2 \%$ of Kenyan, Brazilian and Iranian hearts [7, 28], and in $60.5 \%$ of Pakistanian hearts [13].

The dominance pattern of the coronary circulation has a great clinical importance, where a significant correlation was reported between the coronary dominance on one hand and the incidence and prognosis of the coronary artery diseases on the other hand. The right coronary dominance revealed a significant association with coronary occlusive disease and RCA lesion [9]. Though right coronary dominant circulation was the most prevalent pattern in most studies, the incidence of coronary artery diseases, cardiogenic shock and isolated aortic stenosis were more frequently recorded among patients with left or balanced coronary dominance compared to those with right coronary dominant circulation [13]. Moreover, left coronary dominance was found to have significantly higher mortality than right and balanced dominances. Lesion of AIA would have more profound clinical importance in a left dominant heart than in a right dominance, where dominance had a role in AIA stenosis [11]. These results were in disagreement with the findings reported by Balci and Yilmaz [7], who stated that the dominancy of coronary circulation does not predispose to coronary atherosclerosis. Moreover, the dominance coronary circulation plays an important role in the inferior infarcts of the heart. Although the inferior wall infarcts were less extensive than anterior infarcts, they were more important as they can cause various degrees of atrioventricular block in approximate $30 \%$ of cases. The dominant RCA usually supplies the atrioventricular node. Therefore the inferior wall infarcts caused by occlusion of the RCA will have higher risk of atrioventricular nodal block [1].

Most of the researches did not study sex differences in the incidence of the dominant coronary circulation. In the present study, the incidence of 3 different types of the coronary circulation revealed no significant difference between male and female hearts with dominant right coronary circulation in 
each. However, balanced type of the coronary circulation was the dominant one in both genders with no significant differences between left and right dominant types [14].

The correlation between the presence of MB and the type of coronary circulation was rarely investigated in literature $[6,20]$. In the present study, MB were found in 27 out of $60(45 \%)$ hearts, with no sex difference. Most of them was seen in the hearts of right dominant circulation (52.9\%), followed by the left dominant hearts $(40 \%)$ and then the balanced dominant hearts (31.3\%), however from $77.8 \%$ of MB were observed on the branches of LCA with no significant difference regarding the types of coronary circulation and from $22.2 \%$ of MB were seen over the branches of RCA with a higher incidence in the right dominant circulation. In accordance with the results of the present study, a high incidence of MB was seen over the LCA in case of right coronary dominance [6]. The authors added that in such cases the obstruction of LCA by MB could be overcome by the wider distribution of RCA with less incidence of myocardial suffering and infarcts. Moreover, the obstruction of the dominant artery by the effect of MB could result in bad effect due to larger mass of myocardium being supplied by this dominant artery. However, in disagreement with the results of the present study, a positive correlation was observed between the presence of $\mathrm{MB}$ and the dominant coronary circulation, especially in the left coronary circulation. This correlation was explained on the embryological basis, as there was a developmental pattern between left coronary dominance and MB over the left coronary circulation [20].

Although MB were considered benign or normal variant of the heart vasculature, a close association between the presence of MB and CHD, arrhythmia, sudden cardiac death and cardiomyopathy was reported in different clinical and angiographic studies $[4,22]$. The direct compression of the musculature of $\mathrm{MB}$ on the arterial segment under MB and enhancement of the natural progression of the coronary atherosclerosis in the arterial segment proximal to $M B$ were 2 main mechanisms of $C H D$ in presence of $M B$, as they induced a change in the haemodynamic stress by the force of $\mathrm{MB}$ contraction through a combination of anatomic properties such as length, site, number and thickness $[4,15,22]$. Such abnormal haemodynamic might in part lead to reduction of the blood flow reserve for peripheral perfusion and decrease the diastolic period with production of myocardial ischaemia [4].

The compression effect of MB to the coronary blood flow on the underlying segment was overcome by luminal dilatation and intimal thickening of the proximal segment to $M B[4,6,17]$. These findings were concomitant with those of the present study, where the external diameter of the pre-bridge segment of AIA, DA and PIA was $3.1 \pm 0.16 \mathrm{~mm}, 2.5 \pm$ $\pm 0.13 \mathrm{~mm}$ and $2.56 \pm 0.24 \mathrm{~mm}$ and that of the postbridge segments was $2.9 \pm 0.16 \mathrm{~mm}, 2.4 \pm 0.13 \mathrm{~mm}$, $2.4 \pm 0.18 \mathrm{~mm}$, respectively. Also, thicker intima and wider lumen were noted in the segment of AIA proximal to $M B$ as compared to the parts under and distal to MB. In agreement with the results of the present study, $23.05 \%$ reduction of the arterial lumen was recorded during the passage under MB from pre-bridge to post-bridge course, where the arterial calibre just before the $M B$ was $2.17 \pm 0.16 \mathrm{~mm}$, $1.92 \pm 0.55 \mathrm{~mm}$ at a mid-point and $1.67 \pm 0.57 \mathrm{~mm}$ just after MB [5]. Moreover, the comparison of the external diameter of the AIA under the MB with that of the segments just before and after the MB revealed that the external diameter of the segment proximal to $M B$ was $2.76 \pm 0.76 \mathrm{~mm}$, while that of the segments under $\mathrm{MB}$ and distal to $\mathrm{MB}$ were $2.08 \pm 0.54 \mathrm{~mm}$ and $1.98 \pm 0.59 \mathrm{~mm}$, respectively [19]. The difference in the diameters between proximal and distal segments to $\mathrm{MB}$ induced a disturbance in blood flow with high wall stress. These factors contributed to the development of atherosclerosis in the proximal segment of the MB [15, 22]. Clinical diagnosis of MB must be considered in patients with angina symptoms, in absence of the risk factors or evidence of ischaemia [24]. Thus, knowledge of mechanical and histological effects of MB is essential to determine pathophysiology of atherosclerotic changes in hearts with ischaemic manifestations without an obvious aetiological factor.

In agreement with the findings of the present study, the main diameter of the intramuscular segments was $2 \pm 1.8 \mathrm{~mm}$ and $1.5 \pm 0.6 \mathrm{~mm}$ for AlA and the remaining arteries respectively, while the diameter of the proximal segments was significantly larger than that of the intramuscular segments, being $2.8 \pm 0.5 \mathrm{~mm}$ for AlA and $1.9 \pm 0.3 \mathrm{~mm}$ for the remaining arteries [18]. In addition to the reduction of the diameter of the intramuscular segments, structural differences between intramuscular and epicardial segments were noted as well [19]. Marked reduction of 
the intimal thickness and changes in the endothelial cell morphology of the intima were observed in the tunnelled segment of the coronary branch. These changes might be due to the exposure of intima to a high shear stress [10]. Histologically, the intima of the bridged segment was formed only of contractile-type smooth muscle cells and an abundance of interstitial spiralled collagen, with the absence of the synthetic-type of smooth muscle cells that proliferate and produce the collagen and elastic fibres in the intima as atherosclerosis progresses [2]. Similar findings were observed in the present study. This structure suggests a negative correlation between the MB and atherosclerosis.

In the present study, a relative thickening in the tunica intima with duplication of internal elastic lamina and wider lumen were observed in the arterial segments proximal to $\mathrm{MB}$ comparing to those of both bridged and distal arterial segments to MB. These findings were in agreement with that reported in previous studies [28] and might provide an explanation to the higher incidence of atherosclerosis in proximal arterial segment to MB with protection to the bridged segment [22]. The concentration of elastic fibres in the proximal and distal segments to MB might be related to more haemodynamics in these segments than mere atherosclerosis [28].

In the present study, wide perivascular space containing an excessive amount of connective tissues was seen in the bridged arterial segments comparing to those of both proximal and distal segments to MB. Similarly, a distinct perivascular spaces were noted all around the bridged segment of the coronary artery. The adipose tissue within this space might constitute a coronary cushion against the compressive forces on the bridged vessels during the systole [28]. Moreover, the adipose tissue within the perivascular space may be involved in local stimulation of atherosclerotic evolution through active functions of proinflammatory cytokines and adipokines secreted from this adipose tissue. However, the authors added that the adipokines source was a controversy whether they derived from the blood within the coronary arteries or from adipose tissues, where an absence of atherosclerosis in coronary artery beneath MB was caused by the lack of adipose tissue around this segment [23]. However, there was no significant association between the adipose tissue ratio of perivascular space beneath the $\mathrm{MB}$ with atherosclerosis suppression ratio, where the adipose tissue neither functions as a coronary cushion against MB compression nor was associated with the atherosclerosis development [16]. The authors added that large amount of fibrosis in MB muscle may reduce the compressive force on the bridged artery, but they could not find a significant association between MB fibrosis rate and the atherosclerosis suppression ratio.

The intramural coronary arteries showed thinner wall than the epicardial coronary arteries. The reason of this was the difference in the mural stress, whether linear (shear) or circumferential (hoop). This simple difference could decrease the propensity for arteriosclerosis of mural coronary artery, because the thinner walls allowed more oxygen and nutrients to diffuse to supply the intima, media and adventitia. A direct correlation between the mural stress and development of atherosclerosis was reported [3]. The authors added that in epicardial coronary arteries by increase of the thickness of the arterial wall of more than $0.035 \mathrm{~mm}$, the vasa vasorum begins to develop. The epicardial coronary arteries were invested by adipose tissue, while the intramural arteries were surrounded by the myocardium of MB. Similar findings were observed in the segments of the AIA of the present study.

The intima-media ratio in presence or absence of $\mathrm{MB}$ in patients with MI was previously studied [15]. The authors revealed that the intima-media ratio was usually 0.2 to 0.8 in almost normal AIA and $<1.5$ in AIA segments that had diffuse intimal thickening or mild eccentric raised lesions. Moreover, in cases with $M B$, the intima-media ratio beneath the MB was significantly lower than that in the segments proximal and distal to the MB, while the ratio of severe intimal lesions in proximal segments was higher in patients with MI than in cases with no MI. Meanwhile, in patients having $\mathrm{MI}$ with presence of $\mathrm{MB}$, the intimamedia ratio beneath the MB was significantly lower than that proximal to $M B$ and the ratio distal to the $M B$ was also lower than that proximal to the MB.

As a result of the association between $M B$ and atherosclerosis is controversial (remains unclear), different studies were performed to explain this point. In a few specific studies, thinning of the arterial wall, absence of vasa vasorum and embryological origin of smooth muscle cells on the arterial segment under MB were supposed to be the protective factors against the incidence of atherosclerosis [3], 
while in others the mechanical compression of MB on the underlying arterial segments was supposed to be the main factor for the higher incidence of atherosclerosis in the arterial segments proximal to the $M B$, where the contraction of the muscle fibres of the MB induced an alteration in the haemodynamic force and increased shear stress in the arterial segment under MB. Such higher shear stress reduced the ability of the intimal smooth muscle cells to produce collagen fibres, changed the shape of the endothelial cell lining the proximal arterial segments to become polygonal and flat, while those under MB were spindle-shape, engorged and aligned in the direction of the blood flow [17]. The differences in shape and alignment of the endothelial cells between the proximal and under segments of $M B$ reduced the susceptibility to atherosclerosis in the arterial segments under MB [15]. Moreover, an absence of atherosclerosis in the coronary segment beneath MB might be in part due to the lack of adipose tissue around the arterial segment beneath MB [23]. Conversely, there was no significant association between the adipose tissue ratio of perivascular space beneath the MB with atherosclerosis suppression ratio [16]. Rare occurrence of atherosclerosis in the arterial segments under and distal to the MB due to a decreased tensile stress, increased endothelial shear stress and decreased wall motion during cardiac cycle [8].

MB are common finding at autopsy of normal subjects with no gender-significant difference. It has been thought to be a congenital, generally benign anatomic variation with an embryological base. MB had different number, length and thickness. Commonly, they were seen on AIA and to a lesser extent on $\mathrm{RCA}$. The presence of MB may predispose to different heart diseases like ischaemia, arrhythmias, ventricular dysfunction and sudden cardiac death due to the higher incidence of atherosclerosis in the segments just proximal to them, whereas the tunnelled segment is relatively spared despite the evidence of endothelial dysfunction with subsequent vasospasm and thrombosis. Wider lumen and thicker wall were observed in the segment just proximal to MB comparing to that of both tunnelled and distal segments. Thus, the knowledge of $\mathrm{MB}$ is essential for cardiologists to detect aetiology of different heart-related problems, to plan the mode of treatment and to predict their prognosis as well.

\section{CONCLUSIONS}

Awareness of the morphological properties and pathological effects of the MB on the epicardial coronary branches can help the clinicians and cardiologists to plan better therapeutic strategies and surgical decision in patients with CHD who have an MB. Also the dominant coronary artery circulation and its clinical importance should be known by cardiologists and be included in their interpretations and decision.

\section{ACKNOWLEDGEMENTS}

Great appreciations to all technical members of the Anatomy Department, Faculty of Medicine, King Abdulaziz University for their help during undertaking this work.

\section{REFERENCES}

1. Amin K, Javed M, Mehmood A, Zakaria M (2004) Acute inferior wall myocardial infarction: frequency of $\mathrm{AV}$ blocks. The Professional, 11: 31-37.

2. Algeria RJ, Herrmann J, Holmes DR Jr, Lerman A, Rihal CS (2005) Myocardial bridging. Eur Heart J, 26: 1159-1168.

3. Botta DM, Elefteriades JA (2009) Why are the intramyocardial portions of the coronary arteries spared from arteriosclerosis? Clinical implications. Int J Angiol, 18: 59-61.

4. Bourassa MG, Butnaru A, Lesperance J, Tardif JC (2003) Symptomatic myocardial bridges: overview of ischemic mechanisms and current diagnostic and treatment strategies. J Am Coll Cardiol, 41: 351-359.

5. Ballesteros LEA, Aristeguieta LMR, Tellez SB (2009) Morphological description and clinical implications of myocardial bridges: An anatomical study in Colombians. Arq Braz Cardiol, 92: 242-248.

6. Bharambe V, Arole V(2008) The study of myocardial bridges. J Anat Soc India, 57: 14-21.

7. Balci B, Yilmaz O (2004) Atherosclerotic involvement in patients with left or right dominant coronary circulation. Kardiol Pol, 60: 564-566.

8. Chatzizisis YS, Giannoglou GD (2009) Myocardial bridges are free from atherosclerosis: Overview of the underlying mechanisms. Can J Cardiol, 25: 219-222.

9. Desch S and Harzendorf C (2011) Prevalence and clinical impact of left coronary dominance in patients with aortic stenosis. j Heart Valve Dis, 20: 23-28.

10. Dermengiu D, Vovalis I, Hostiuc S, Curca GC, Rusu MC, Luca L (2010) Morphological features in myocardial bridging. Rom J Leg Med, 18: 163-170.

11. Das H, Das G, Das DC, Talukdar K (2010) A study of coronary dominance in the population of Assam. J Anat Soc India, 59: 187-101.

12. Fazliogullari Z, Karabulut AK, Kayrak M, Uysal I, Dogan N, Altunkeser BB (2010) Investigation and review of myocardial bridges in adult cadaver hearts and angiographs. Surg Radiol Anat, 32: 437-445.

13. Fazlul AM, Malik SN, Khan IS, Kachlu AR, Rehman M, Hussain J, Ahmad I (2011) Coronary artery dominance: 
what pattern exists in Pakistani Population. Ann Pak Inst Med Sci, 7: 3-5.

14. Gawlikowska-Sroka A, Miklaszewska D, Czerwinski F (2010) Analysis of the influence of heart size and gender on coronary circulation type. Folia Morphol, 69: 35-41.

15. Ishikawa Y, Akasaka Y, Suzuki K, Fujiwara M, Ogawa T, Yamazaki K, Niino H, Tanaka M, Ogata K, Morinaga S, Ebihara Y, Kawahara Y, Sugiura H, Takimoto T, Komatsu A, Shinagawa T, Taki K, Satoh H, Yamada K, Yanagida-lida M, Shimokawa R, Shimada K, Nishimura C, Ito K, Ishii T (2009) Anatomic properties of myocardial bridge predisposing to myocardial infarction. Circulation, 120: 376-383.

16. Iuchi $A$, Ishikawa $Y$, Akishima-Fukasawa $Y$, Fukuzawa $R$, Akasaka $Y$, Ishii T (2013) Association of variance in anatomical elements of myocardial bridge with coronary atherosclerosis. Atherosclerosis, 227: 153-158.

17. Kim PJ, Hur G, Kim SY, Namgung J, Hong SW, Kim YH, Lee WR (2009) Frequency of myocardial bridges and dynamic compression of epicardial coronary arteries: a comparison between computed tomography and invasive coronary angiography. Circulation, 119: 1408$-1416$.

18. Konen E, Goitein O, Sternik L, Eshet Y, Shemesh J, Segni $E$ (2007) The prevalence and anatomical patterns of intramuscular coronary arteries. A coronary computed tomography angiographic study. J Am Coll Cardiol, 49: 587-593.

19. Lima VJ, Cavalcanti JS, Tashiro T (2002) Myocardial bridges and their relationship to the anterior interventricular branch of the left coronary artery. Arq Bras Cardiol, 79: 215-222.
20. Loukas M, Curry B, Bowers M, Louis RG Jr, Bartczak A, Kiedrowski M, Kamionek M, Fudalej M, Wagner T (2006) The relationship of myocardial bridges to coronary artery dominance in adult human heart. J Anat, 209: 43-50.

21. Lozano I, Baz JA, Lopez Palop R, Pinar E, Pico F, Valdez M, Larman M, Marinez Ubago JL (2002) Pronostico a largo plazo de los pacientes con trayecto intramiocardico de la arteria descendente anterior con compression sistolica. Rev Esp Cardiol, 55: 359-364.

22. Möhlenkamp S, Hort W, Ge J, Erbel R (2002) Update on myocardial bridging. Circulation, 106: 2612-2622.

23. Payne GA, Kohr MC, Tune JD (2012) Epicardial perivascular adipose tissue as a therapeutic target in obesity-related coronary artery disease. Br J Pharmacol, 165: 659-669.

24. Periera AB, Castro DA, Menegotto ET, Amaral WM, Castro GS (2010) Myocardial bridging: therapeutic and clinical development. Arq Bras Cardiol, 94: 175-181.

25. Reese E David, Takashi Mikawa, David M Bader (2002) Development of the coronary vessel system. Circulation Res, 91: 761-768.

26. Sousa-Rodrigues CF, Alcantara F, Buarque LC, da Rocha AC, Alencar Sliva R, Olave E (2006) Aspectos anatomicos y biometricos de los puentes de miocardio y sus relaciones con la arteria interventricular anterior venas adyacentes. Int J Morphol, 24: 270-284.

27. Schlesinger MJ (1940) Relation of anatomic pattern to pathologic conditions of the coronary arteries. Arch Path, 30: 403-415.

28. Saidi HS, Ongeti WK, Ogeng'o J (2010) Morphology of human myocardial bridges and association with coronary artery disease. African Health Science, 10: 242-247. 